\title{
New Extra Large Pore Chromium Oxophenylphosphate: An Efficient Catalyst in Liquid Phase Partial Oxidation Reactions
}

\author{
Nabanita Pal, Manidipa Paul, and Asim Bhaumik
}

Department of Materials Science, Indian Association for the Cultivation of Science, Jadavpur, Kolkata - 700 032, India

\begin{abstract}
A new supermicroporous chromium oxophenylphosphate (CPP-1) has been synthesized using phenylphosphonic acid (PPA) as phosphorus source under hydrothermal condition at $443 \mathrm{~K}$ without using any structuredirecting agent (SDA). The material has been characterized by powder XRD, FE SEM-EDS, TEM, TG-DTA, $\mathrm{N}_{2}$ sorption, XPS, FT IR, UV-Vis spectroscopic techniques and CHN chemical analysis. CPP-1 showed specific surface area of 313 $\mathrm{m}^{2} \mathrm{~g}^{-1}$ with extra large supermicropores having dimension of $c a .1 .4 \mathrm{~nm}$. TEM result suggested lamellar type pore-wall structure for this material. It exhibited good catalytic property in partial oxidation of olefins in presence of tertbutylhydroperoxide (TBHP) as oxidant.
\end{abstract}

Keywords: Chromium oxophenylphosphate, lamellar, organic-inorganic hybrid material, partial oxidation, supermicropores.

\section{INTRODUCTION}

The chemistry of chromium has occupied a special place in fundamental research due to the redox behavior and remarkable stability of different oxidation states [1]. Cr (III)containing complexes and materials are used extensively in different oxidation reactions as catalyst to obtain value added organic fine chemicals [2]. Chromium phosphates synthesized previously, exhibit wide application in ion exchange [3], catalytic reactions including oxidative dehydrogenation [4], acid catalyzed reactions [5], etc. However, these phosphate-based catalysts have several drawbacks in large-scale industrial applications due to very low specific surface areas and as a consequence low turn over frequencies.

On the other hand, microporous [6-8] and mesoporous materials [9-12] have attracted major research interests in catalysis [13], selective adsorption [14], and ion exchange [15] etc. due to their very high internal surface areas and tunable narrow pore size distributions. With the discovery of silica based organic-inorganic hybrid microporous and mesoporous materials [16-20]; the importance of this field is increased in greater extent due to possibility of grafting a large number of organic moieties into the parent inorganic structure. Introduction of various organic functionalities in the silica framework enhance the flexibility, as a result of which the physical and chemical properties of the materials change totally. These hybrid porous solids show widespread application in catalysis [21], chemosensor [22], environmental clean-up [23], optoelectronics [24], magnetic materials [25], light harvesting [26] and so on. Incidentally, all these research works are mainly focused on the silicabased mesostructure. Comparatively, a minor attention is given to hybrid inorganic components like phosphate based

*Address correspondence to this author at the Department of Materials Science, Indian Association for the Cultivation of Science, Jadavpur, Kolkata - 700 032, India; Fax: +91-33-2473-2805;

E-mail: msab@iacs.res.in hybrid materials [27, 28]. Several porous metallophosphates with application in selective organic reactions, optical and photocatalysis [29] have been reported recently. Syntheses of these porous phosphate materials require either a single molecule template or supramolecular assembly of surfactants, which can act as structure-directing agent (SDA) [30]. The porosity in the framework is generated after effective removal of the template molecules from the phosphate composites.

However, this templating pathway is not very straightforward and often removal of the template may collapse the mesostructure. Therefore, it is much more advantageous to synthesize porous phosphate-based materials without the aid of any SDA. To data, there is no report of synthesis of porous chromium phosphate material without using any template. Herein, for the first time, we report a template-free synthesis of a supermicroporous chromium oxophenylphosphate (CPP-1). The synthesized material shows high surface area and excellent activity in selective liquid phase partial oxidation of olefins using TBHP as oxidant.

\section{EXPERIMENTAL SECTION}

\section{Method}

CPP-1 has been synthesized using phenylphosphonic acid (Aldrich) and chromium (III) chloride (Loba Chemie) as phosphorus and chromium sources, respectively. In a typical synthetic procedure, $0.80 \mathrm{~g}$ phenylphosphonic acid (PPA) was first dissolved in $35 \mathrm{~g}$ water. Then $1.34 \mathrm{~g}$ chromium (III) chloride was dissolved in $5 \mathrm{~g}$ water and added to the PPA solution dropwise. Resulting green mixture was stirred for 2 $\mathrm{h}$. The $\mathrm{pH}$ of the synthetic mixture was $c a .1 .0$. Then tetramethylammonium hydroxide $(25 \%$ aqueous solution, Aldrich) was added to the mixture to increase the $\mathrm{pH} c a$. 4-5 until a thick green precipitate appeared. It was stirred for another $3 \mathrm{~h}$ at room temperature. The final synthetic gel was autoclaved at $443 \mathrm{~K}$ for $24 \mathrm{~h}$. The molar ratio of the final mixture was PPA: $\mathrm{CrCl}_{3}: \mathrm{H}_{2} \mathrm{O}=1: 1: 445$. The product was 
filtered, washed with water and dried under vacuum at ambient temperature.

\section{Characterization Tools}

Powder X-ray diffraction patterns of the materials were recorded on a Bruker AXS D-8 Advance diffractometer operated at $40 \mathrm{kV}$ voltage and $40 \mathrm{~mA}$ current and calibrated with a standard silicon sample, using Ni-filtered $\mathrm{Cu} \mathrm{K}_{\alpha}(\lambda=$ $0.15406 \mathrm{~nm})$ radiation. Nitrogen adsorption/desorption isotherms were obtained using a Bel Japan Inc. Belsorp-HP at $77 \mathrm{~K}$. Before the measurement, the sample was degassed at $393 \mathrm{~K}$ for $12 \mathrm{~h}$. TEM images were recorded in a Jeol JEM 2010 transmission electron microscope. JEOL JEM 6700F field emission scanning electron microscope with an energy dispersive X-ray spectroscopic (EDS) attachment was used to record SEM images of the sample and its surface chemical composition. Thermogravimetry (TG) and differential thermal analysis (DTA) were carried out in a TA Instruments thermal analyzer TA SDT Q-600. FT IR spectrum of the sample was recorded on $\mathrm{KBr}$ pellets by using a Nicolet MAGNA-FT IR 750 spectrometer series II. UV-Visible diffuse reflectance spectra were obtained by using a Shimadzu UV 2401PC spectrophotometer with an integrating sphere attachment and $\mathrm{BaSO}_{4}$ pellet was used as background standard. Carbon, hydrogen and nitrogen contents were analyzed using a Perkin Elmer 2400 Series II $\mathrm{CHN}$ analyzer. X-ray photoelectron spectra (XPS) of the CPP-1 sample were recorded in VG Microtech. Clam 2 Analyzer, with Mg K-alpha X-ray source. The spectrum was calibrated taking the reference of $\mathrm{C} 1 \mathrm{~s}$ peak at $284.5 \mathrm{eV}$.

\section{Catalytic Conditions}

The liquid phase partial oxidation reactions of styrene, cyclohexene and allyl alcohol were carried out taking $0.5 \mathrm{~g}$ of the substrate with same molar ratio of tertbutylhydroperoxide (TBHP, 70\%, Aldrich) in $8.0 \mathrm{ml}$ acetonitrile solvent containing required amount of the CPP-1 sample (20 wt $\%$ of the substrate). The reactions were performed in a round-bottomed flask fitted with a condenser and temperature-controlled oil bath on a magnetic stirrer. The temperature was maintained at $333 \mathrm{~K}$ and the reaction products were collected at regular intervals. The progress of the reaction was monitored by an Agilent 4890D gas chromatograph (FID detector) fitted with a capillary column; $0.5 \mathrm{ml} n$-heptane was introduced to each of the reaction mixtures as internal standard before analysis.

\section{RESULTS AND DISCUSSION}

\section{Chemical Analysis}

The chemical composition of the synthesized chromium oxophenylphosphate sample was determined by $\mathrm{CHN}$ analysis and it was further verified from EDS and XPS data. $\mathrm{CHN}$ analysis suggested the presence of $30.2 \% \mathrm{C}$ and 2.65 $\% \mathrm{H}$ with ratio $\mathrm{C} / \mathrm{H}=11.4$ in this sample. From $\mathrm{EDS}$ data (Table 1) the molar ratio of $\mathrm{P} / \mathrm{Cr}$ was 1.34 and that of $\mathrm{C} / \mathrm{O}$ was 1.51 . The mole ratio of $\mathrm{P} / \mathrm{Cr}$ calculated from XPS data was observed 1.2. Thus ratio of oxygen to hydrogen calculated was $\mathrm{O} / \mathrm{H}=7.55$. Hence the ratio $\mathrm{C}: \mathrm{H}: \mathrm{O}=11.4$ : 1: 7.5 was almost identical to the $\mathrm{C}: \mathrm{H}: \mathrm{O}=11: 1.1: 7.4$ in phenylphosphonic acid. Little excess of oxygen was present in CPP-1. This could be due to the oxophenylphosphate framework structure.
Table 1. EDS surface Chemical Analysis of CPP-1

\begin{tabular}{|c|c|c|c|c|}
\hline \multicolumn{2}{|c|}{ Element } & (keV) & Mass \% & Atomic \% \\
\hline $\mathrm{C}$ & $\mathrm{K}$ & 0.277 & 42.74 & 55.56 \\
\hline $\mathrm{O}$ & K & 0.525 & 37.70 & 36.80 \\
\hline $\mathrm{P}$ & K & 2.013 & 8.66 & 4.37 \\
\hline $\mathrm{Cr}$ & K & 5.411 & 10.89 & 3.27 \\
\hline \multicolumn{2}{|c|}{ Total } & & 100.00 & 100.00 \\
\hline
\end{tabular}

\section{Characterizations}

\section{Nanostructure: Powder XRD, TEM and FE SEM}

The powder X-ray diffraction pattern of the CPP-1 sample is shown in Fig. (1). An intense peak at $2 \theta=5.89(d$ $=1.49 \mathrm{~nm}$ ) was observed suggesting an interlayer distance of $1.49 \mathrm{~nm}$. This figure further indicated existence of many small diffraction peaks with multiples of $2 \theta=5.89$. This suggests the lamellar type structure of the chromium oxophenylphosphate material. Multilamellar structural feature of the material is also seen from the transmission electron micrograph (TEM) of the sample as shown in Fig. (2, up). The low electron density spots with uniform diameter correspond to the micropores of $c a .1 .38 \mathrm{~nm}$ in the specimen. Thus, powder XRD and TEM data suggested that CPP-1 has lamellar type structure with supermicropores [31,32] of dimension ca. $1.4 \mathrm{~nm}$. Fig. (2, down) shows FE SEM image of the sample. This image suggests the formation of small spherical particles of 10-15 nm in size and these are found to form relatively large spherical aggregates in some places of the specimen. These tiny porous spherical particles are significant from adsorption and catalytic points of view.

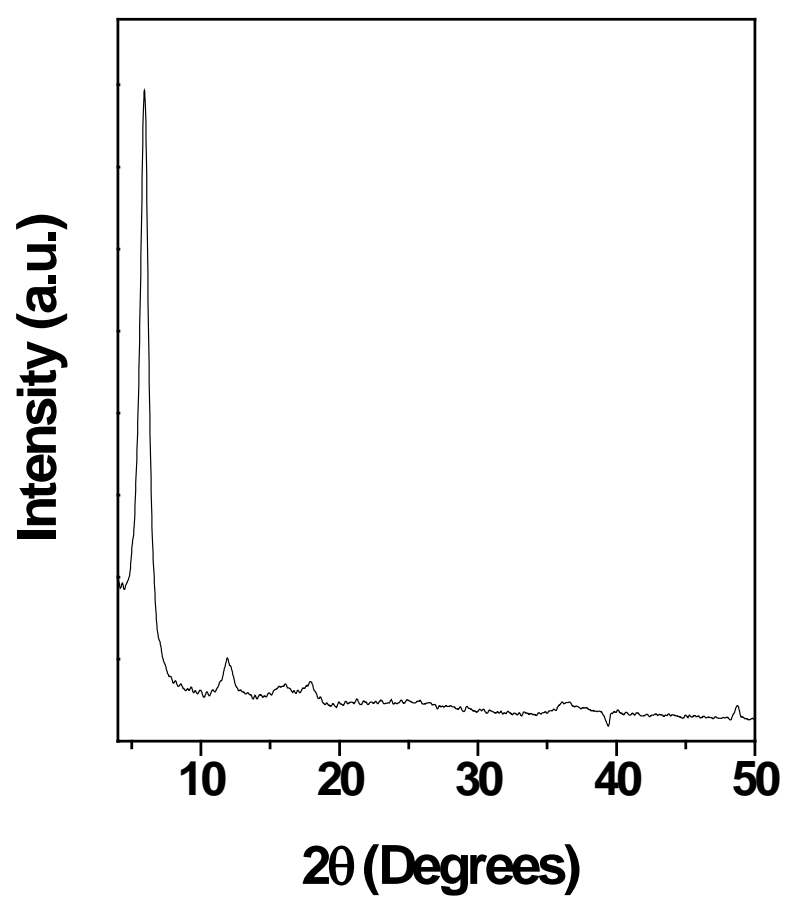

Fig. (1). Powder XRD patter of CPP-1. 


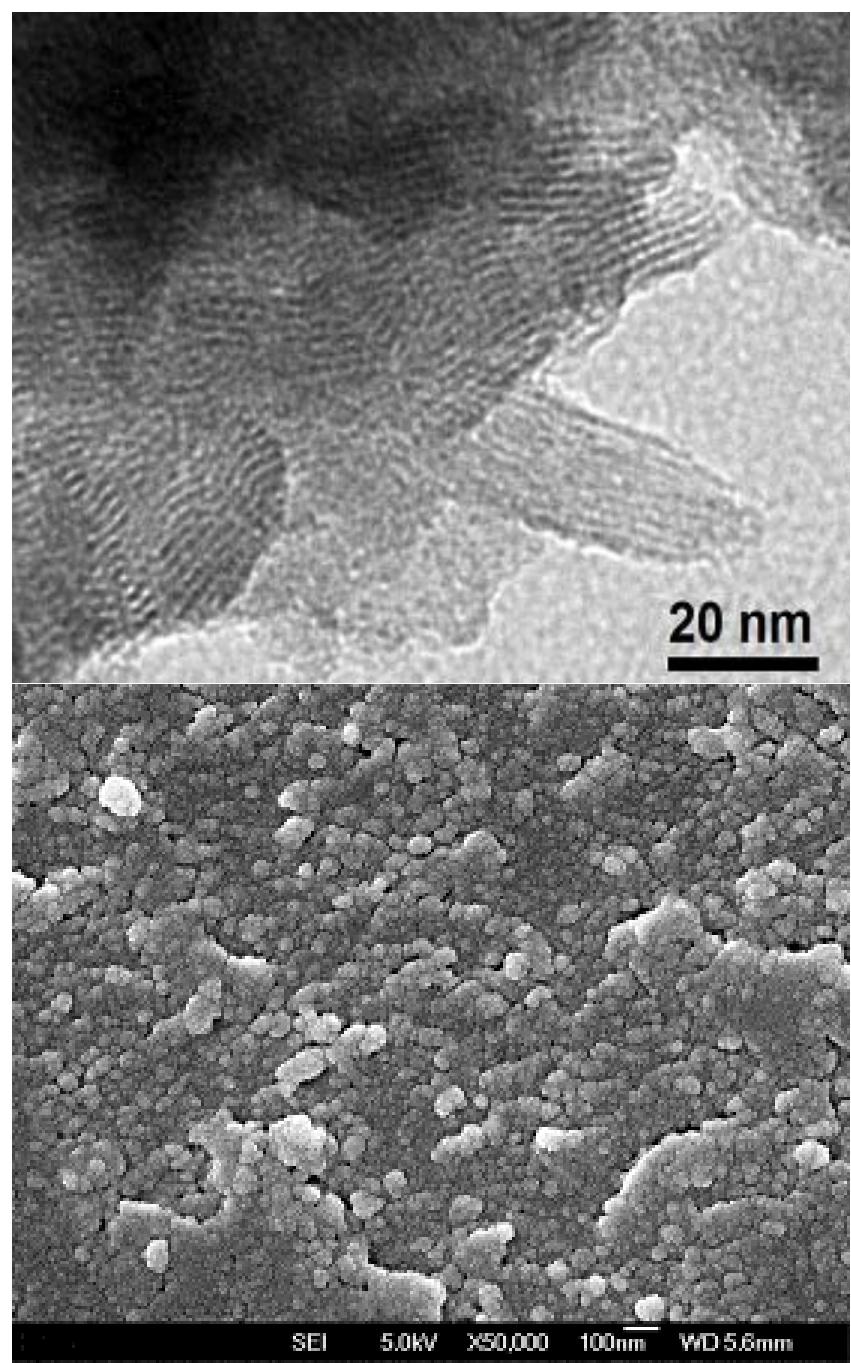

Fig. (2). Up: TEM image of the as-synthesized CPP-1. Down: FE SEM image of the CPP-1.

\section{$\mathbf{N}_{2}$ Adsorption Study}

$\mathrm{N}_{2}$ adsorption/desorption isotherm of the sample CPP-1 is shown in Fig. (3). This sample showed the features of both type I and IV isotherms with hysteresis loop at high $\mathrm{P} / \mathrm{P}_{0}$ regions $[33,34]$. It suggests the presence of very large micropores/supermicropores. The BET surface area and pore volume of the hybrid sample were $313 \mathrm{~m}^{2} \mathrm{~g}^{-1}$ and $0.25 \mathrm{~cm}^{3} \mathrm{~g}^{-1}$. The pore diameter estimated using the NLDFT method [35] is shown in the inset of this figure. Here $\mathrm{N}_{2}$ adsorption at 77 $\mathrm{K}$ on carbon was used as the DFT kernel (slit pore, NLDFT equilibrium model). The bimodal pore size distribution (PSD) suggested the existence of large micropores of $c a$. $1.38 \mathrm{~nm}$. Less intense peak near $c a .4 .1 \mathrm{~nm}$ might be due to the interparticle pores. The micropore diameter estimated from $\mathrm{N}_{2}$ sorption agrees well with that obtained from TEM image analysis. Energy dispersive X-ray spectroscopic (EDS) pattern of a homogeneous large specimen is shown in Fig. (4). As seen from this spectral pattern, all major elements $\mathrm{C}, \mathrm{O}, \mathrm{P}$ and $\mathrm{Cr}$ are present in $\mathrm{CPP}-1$ and their composition agree well with the chromium oxophenylphosphate structure as was observed previously for titanium oxophenylphosphate synthesized by using PPA as $\mathrm{P}$ precursor [36].

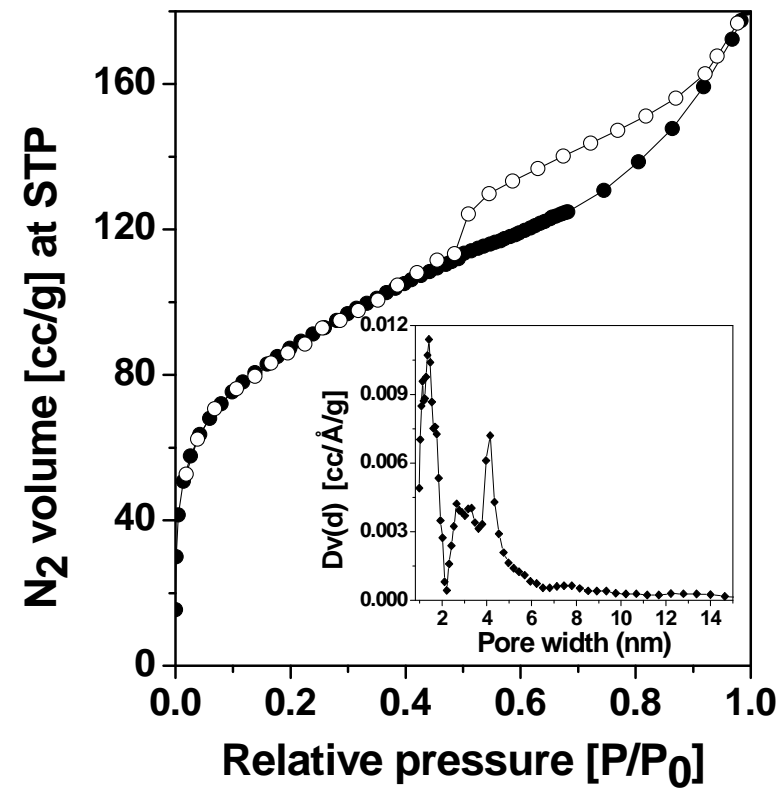

Fig. (3). $\mathrm{N}_{2}$ adsorption (•)-desorption (०) isotherms of CPP-1. Pore size distribution is shown in the inset.

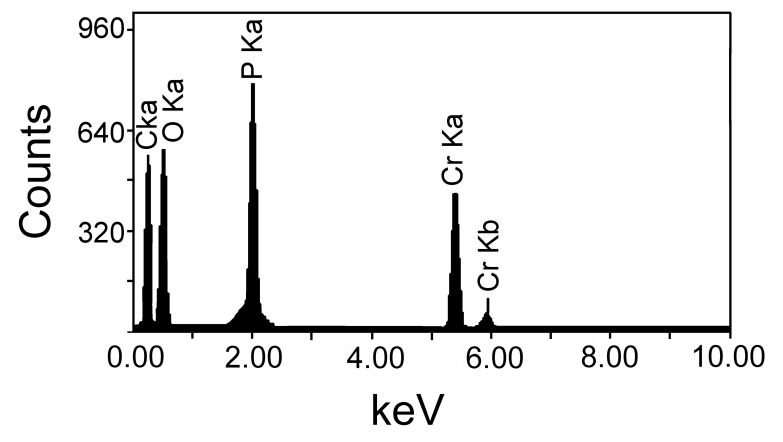

Fig. (4). EDS pattern of CPP-1.

\section{FT-IR and UV-Vis Spectroscopic Analysis}

Incorporation of phenyl group in this mesoporous framework was verified from spectroscopic data. FT-IR spectrum of the hybrid sample is shown in Fig. (5). The spectrum exhibits a peak near $3500 \mathrm{~cm}^{-1}$ and a weaker peak near $1625 \mathrm{~cm}^{-1}$, these correspond to the $\mathrm{O}-\mathrm{H}$ stretching and $\mathrm{H}_{3} \mathrm{O}^{+}$bending vibration, respectively [3,37]. Presence of phenyl group intact with $\mathrm{P}$ atom is indicated by the aromatic C-H vibration at $3062 \mathrm{~cm}^{-1}$ and $2855 \mathrm{~cm}^{-1}$ along with a C-P vibration near $1150 \mathrm{~cm}^{-1}$. Further, the other fingerprint bands near $687 \mathrm{~cm}^{-1}$ and $1436 \mathrm{~cm}^{-1}$ also confirm the presence of phenyl group in CPP-1 framework [38]. Another two bands near $951 \mathrm{~cm}^{-1}$ and $1036 \mathrm{~cm}^{-1}$ indicate $\mathrm{P}-\mathrm{O}$ stretching frequency [39], whereas $\mathrm{Cr}-\mathrm{O}$ bond vibration is observed near $622 \mathrm{~cm}^{-1}$ [40]. In Fig. (6A) UV-visible diffuse reflectance spectra of CPP-1 (a) and PPA (b) are shown. Pure phenylphosphonic acid showed a broad absorption having maxima near $266 \mathrm{~nm}$ due to chromophoric phenyl group [36], which is still present in hybrid CPP-1. In case of CPP-1 two broad absorption peaks near $610 \mathrm{~nm}$ and $421 \mathrm{~nm}$ may be due to $d-d$ transition of octahedral $\mathrm{Cr}$ (III) i.e. first 
one is for ${ }^{4} \mathrm{~A}_{2 \mathrm{~g}} \rightarrow{ }^{4} \mathrm{~T}_{1 \mathrm{~g}}$ (F) transition and second is for ${ }^{4} \mathrm{~A}_{2 \mathrm{~g}} \rightarrow{ }^{4} \mathrm{~T}_{2 \mathrm{~g}}$ (F) transition. No peak around $280 \mathrm{~nm}$ and 370 $\mathrm{nm}$ indicates the absence of $\mathrm{Cr}$ (VI) species [41]. The band gap energy of the hybrid CPP-1 is given in Fig. (6B). For CPP-1 the observed band gap is $4.38 \mathrm{eV}$. Optical band gap for $\mathrm{Cr}$ (III) oxide varies from 4.7 to $5.0 \mathrm{eV}$ depending on the deposition temperature [42]. In our porous chromium oxophenylphosphate sample CPP-1 apart from Cr-O-P bonding and network, presence of surface defect sites could be responsible for the reduction of the band gap to $4.4 \mathrm{eV}$. It is quite clear from the above spectroscopic results that in the CPP-1 framework, $\mathrm{Cr}$ (III) is surrounded by oxygen atoms some of which are attached to P atoms of PPA groups bearing the phenyl moieties and others as hydroxyl groups (defects).

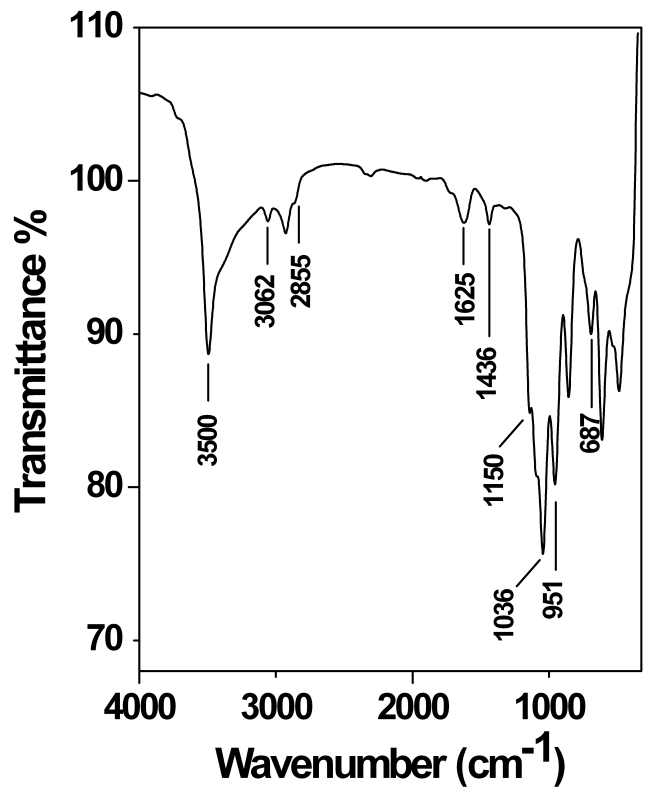

Fig. (5). FT-IR spectrum of CPP-1.

\section{Thermal Analysis}

The TGA (a) and DTA (b) plots of the mesoporous chromium oxophenylphosphate are shown in Fig. (7). The sample exhibited weight loss of $c a .6 \%$ up to $660 \mathrm{~K}$ in the TGA plot. This is due to the removal of physically adsorbed water molecules at the surface. Corresponding broad endotherm in the range of $458-638 \mathrm{~K}$ is observed in the DTA plot. This means the sample is stable up to $660 \mathrm{~K}$ without loss of any organic fragments. At elevated temperature, sharp fall of TGA curve up to $827 \mathrm{~K}$ with further $c a .35 \%$ weight loss indicates the burning of phenyl groups and collapsing of the structure leading to carbonized material. TG analysis was carried out under $\mathrm{N}_{2}$ flow, that's why whole organic mass does not decomposed, but has been converted to carbonized material. This is clearly indicated by the $c a .59$ $\mathrm{wt} \%$ residual mass even after thermal treatment at $1050 \mathrm{~K}$.

\section{XPS Results}

XPS spectra of $\mathrm{C} 1 \mathrm{~s}$ and $\mathrm{O} 1 \mathrm{~s}$ are shown in Fig. (8). C 1s showed one peak with binding energy of $284.5 \mathrm{eV}$ whereas, a peak at $530.9 \mathrm{eV}$ was observed in $\mathrm{O} 1 \mathrm{~s}$ spectrum. C $1 \mathrm{~s}$ suggests the presence of $\mathrm{sp}^{2}$ carbon, i.e. aromatic phenyl group [43] and O 1s peak is for covalent oxygen atom [44]. In Fig. (9A) $\mathrm{Cr} 2 p$ XPS data is shown. The peak position for $\mathrm{Cr} 2 p_{3 / 2}$ was at $577.2 \mathrm{eV}$ and that for $\mathrm{Cr} 2 p_{1 / 2}$ appeared at $586.9 \mathrm{eV}$. Both suggest the presence of $\mathrm{Cr}$ (III) state in the framework [45]. Absence of $2 \mathrm{p}_{3 / 2}$ signal ca. $579.5-580 \mathrm{eV}$ ruled out the possibility of presence of Cr (IV) in the sample [45]. For P 2p XPS data (Fig. 9B) the observed peak at 133.6 $\mathrm{eV}$ implies that pentavalent oxidation state $\mathrm{P}(\mathrm{V})$ is present there i.e. phosphate group remained intact in the material surface [46].
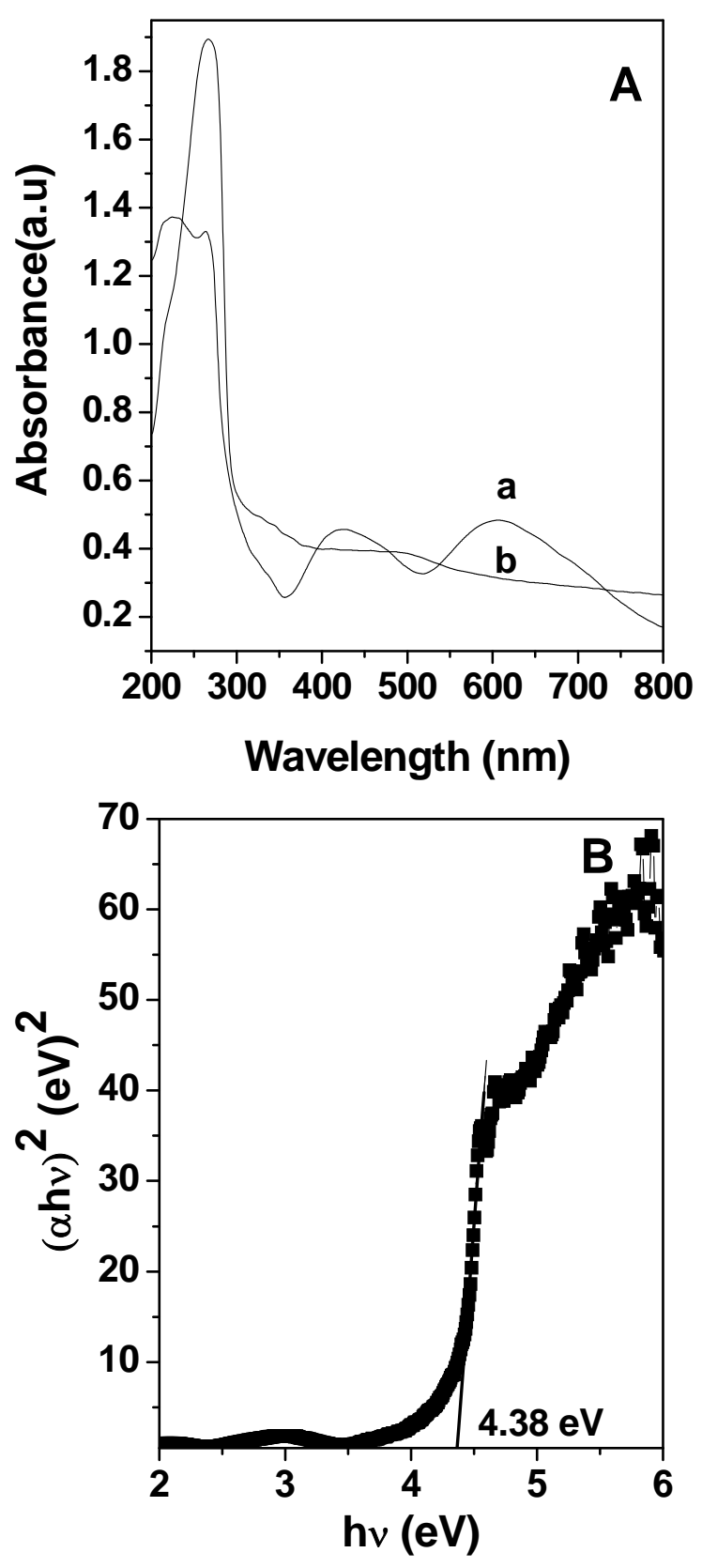

Fig. (6). A: UV-visible diffuse reflectance spectra of CPP-1 (a) and PPA (b). B: Estimated band-gap of CPP-1. 


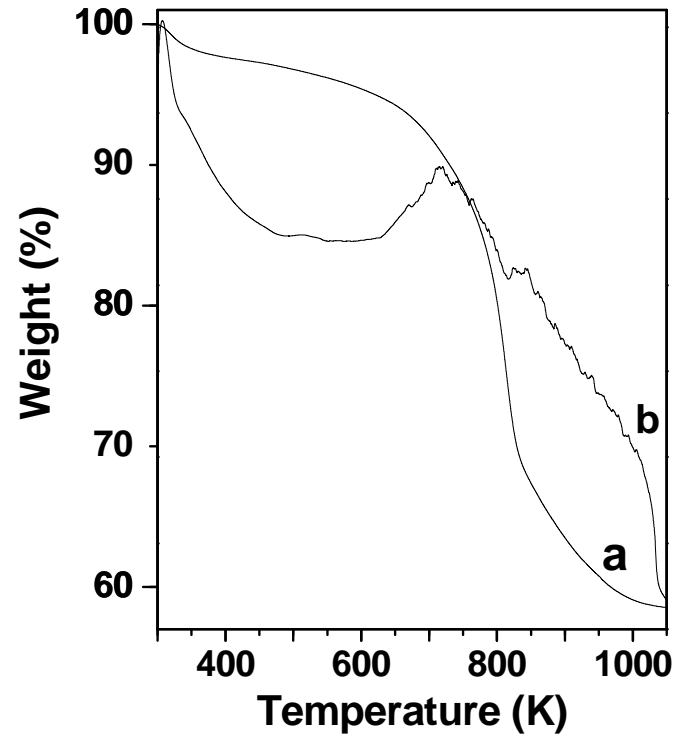

Fig. (7). TG (a) and DTA (b) curves for CPP-1.
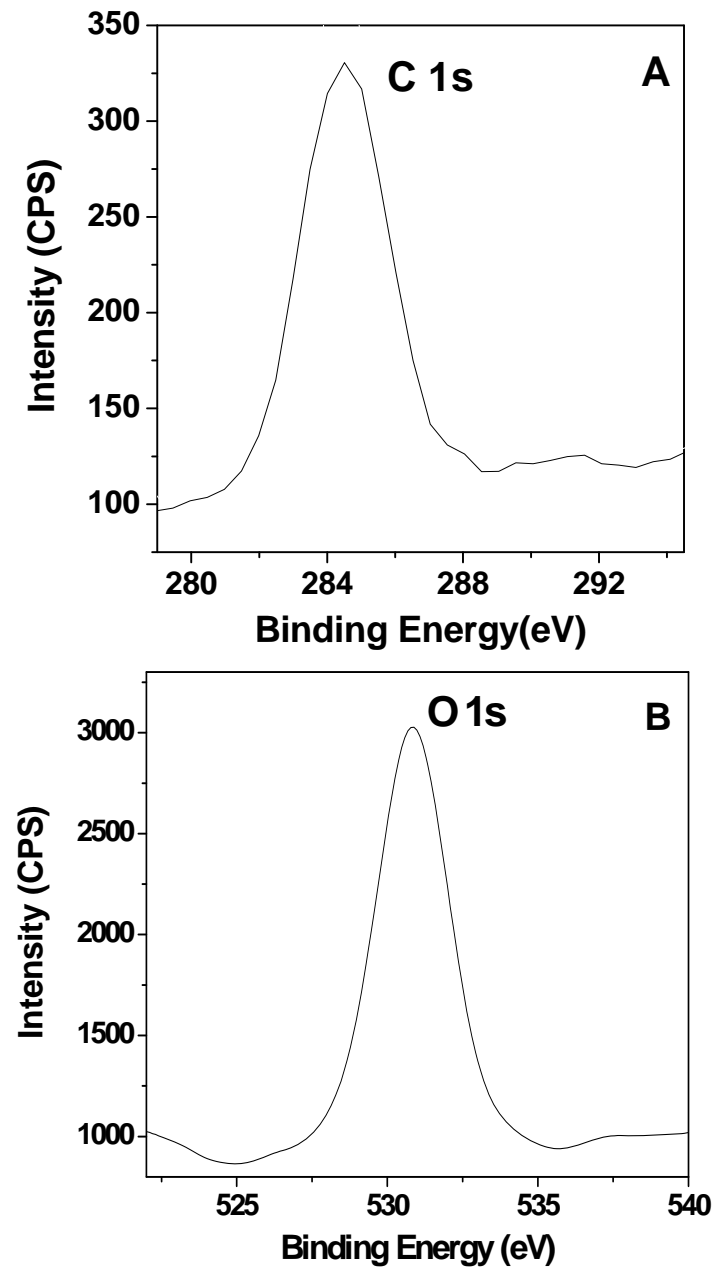

Fig. (8). XPS of C 1s (A) and O 1s (B) for CPP-1.
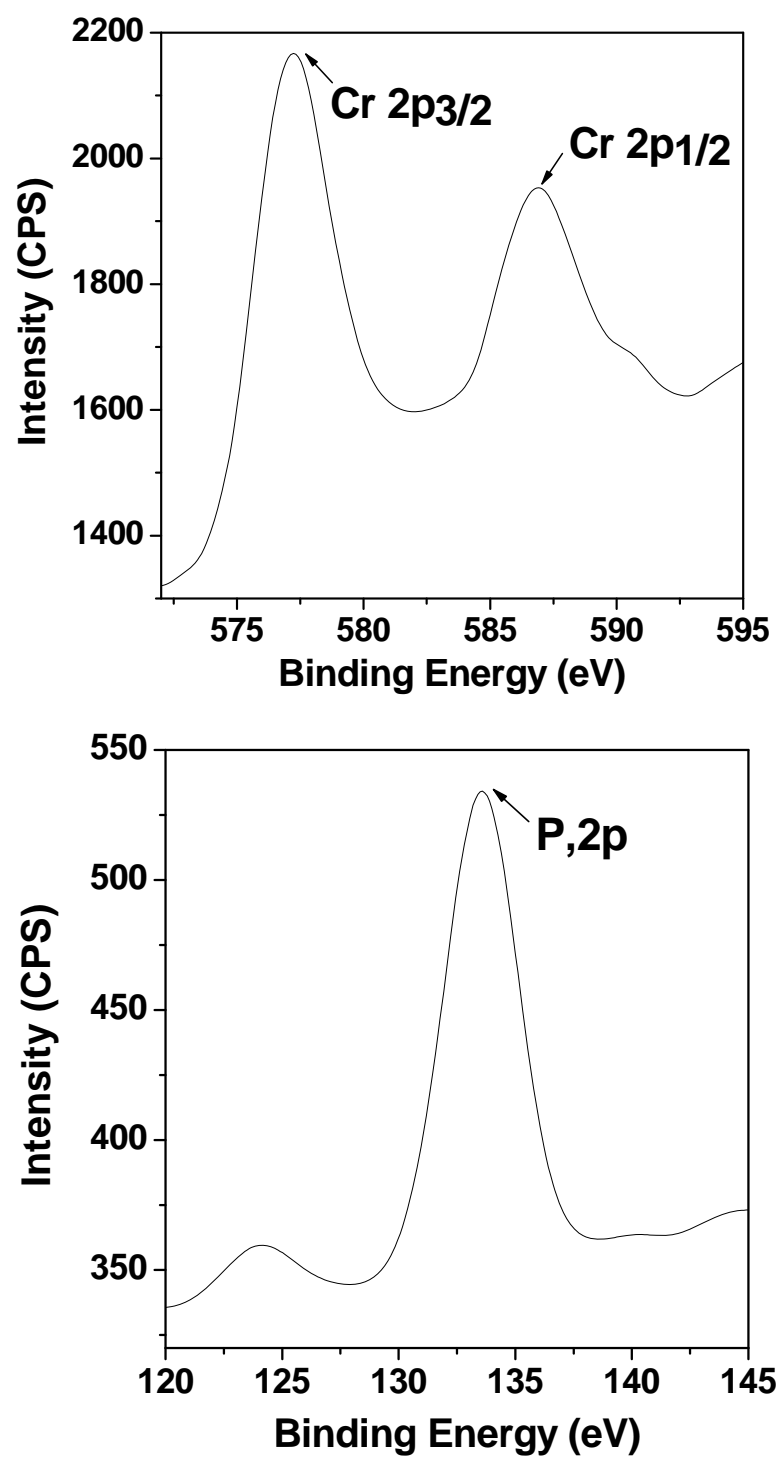

Fig. (9). XPS of Cr $2 p(A)$ and P $2 p(B)$ for CPP-1.

\section{Catalysis}

We have carried out the liquid phase partial oxidation of different olefins in the presence of TBHP as oxidant to explore the catalytic potential of CPP-1 sample. The results are given in Table 2 . For the oxidation of styrene $44 \%$ conversion together with $100 \%$ selectivity for benzaldehyde is observed even after longer reaction time $(24 \mathrm{~h})$. On the other hand for cyclohexene $c a .95 \%$ conversion and high selectivity for cyclohexene-1- ol (ca. 95\%), an allylic oxidation product is observed. In the case of allyl alcohol the products are mainly glycidol (epoxidation product) and glycerol. Latter could form as a result of hydrolysis of the epoxide ring of glycidol in the presence of water in the reaction medium. A blank reaction (Table 2, entry 4 ) on the oxidation of cyclohexene is carried out in absence of any catalyst CPP-1 in the reaction medium, where the reaction could not proceed at all. Our experimental results suggest that these partial oxidation reactions are catalytic in nature [47]. As seen from the table that for all the substrates CPP-1 showed high turn over numbers indicating our new 
Table 2. Liquid Phase Catalytic Reactions Over Mesoporous Chromium Oxophenylphosphate

\begin{tabular}{|c|c|c|c|c|c|}
\hline Substrate & Reaction Time (h) & Conversion (\%) & Products & Product sel. (\%) $^{\text {TON }^{\mathbf{1}}}$ \\
\hline \hline Styrene & 24 & 44.1 & Benzaldehyde & 100 & 33.7 \\
\hline Cyclohexene & 24 & 94.8 & Cyclohexene-1- ol & 94.7 & 91.9 \\
\hline Allyl alcohol & 24 & 31.1 & Glycidol + glycerol & $55.67+44.33$ & 42.6 \\
\hline Cyclohexene $^{2}$ & 12 & 0.6 & - & - \\
\hline
\end{tabular}

${ }^{1}$ Turn over number: moles of substrate converted per mole of $\mathrm{Cr}$.

${ }^{2}$ Reaction was carried out in absence of any catalyst.

supermicroporous chromium oxophenylphosphate is an efficient catalyst in the presence of TBHP as oxidant.

\section{CONCLUSION}

Supermicroporous chromium oxophenylphosphate material having good surface area and high thermal stability has been synthesized hydrothermally, where no structuredirecting agent is used. The new framework has been studied in detail through spectroscopic tools, thermal and chemical analysis along with electron microscopy. Incorporation of phenyl group in this phosphate based molecular sieve network might lead to further functionalization and relevant potential applications. Excellent catalytic reactivity of our porous chromium oxophosphate material in liquid phase partial oxidation of olefins may motivate the researchers to explore their catalytic potential in other liquid phase partial oxidation reactions.

\section{ACKNOWLEDGEMENTS}

AB wishes to thank Department of Science and Technology (DST), New Delhi for financial support. NP and MP are grateful to Council of Scientific and Industrial Research (CSIR), New Delhi for their junior and senior research fellowships, respectively.

\section{ABBREVIATIONS}

$\begin{array}{lll}\text { CPP-1 } & & \text { Chromium oxophenylphosphate } \\ \text { PPA } & & \text { Phenylphosphonic acid } \\ \text { SDA }= & \text { Structure directing agent } \\ \text { TBHP }= & \text { tert } \text {-Butylhydroperoxide } \\ \text { FID }= & \text { Flame ionisation detector } \\ \text { BET }= & \text { Brunauer Emmett Teller } \\ \text { NLDFT }= & \text { Nonlocal density functional theory } \\ \text { REFERENCES } & \end{array}$

[1] Weckhuysen, B. M.; Wachs, I. E.; Schoonheydt, R. A. Surface chemistry and spectroscopy of chromium in inorganic oxides. Chem. Rev., 1996, 96, 3327-3349.

[2] Rao, T. V. M.; Yang, Y.; Sayari, A. Ethane dehydrogenation over pore-expanded mesoporous silica supported chromium oxide: 1 . Catalysts preparation and characterization. J. Mol. Catal. A Chem., 2009, 301, 152-158.

[3] Mustafa, S.; Murtaza, S.; Naeem, A.; Farina, K. Sorption of divalent metal ions on $\mathrm{CrPO}_{4}$. J. Coll. Interf. Sci., 2005, 283, 287293.

[4] Chen, Y. W.; Li, C. P. Chromia-chrominum phosphates as solid acid catalysts. Catal. Lett., 1992, 16, 447-453.

[5] Bautista, F. M.; Campelo, J. M.; Garcia, A.; Luna, D.; Marinas, J. M.; Urbano, J. M. Chromium-aluminium orthophosphates Part 1. Structure, texture, surface acidity and catalytic activity in cyclohexene skeletal isomerization and cumene conversion of CrPO4-AlPO4 catalysts. J. Mater. Chem., 1994, 4, 311-317.

[6] Szostak, R. Molecular Sieves: Principles of Synthesis and Identification; Van Nostrand Reinhold, New York 1989.

[7] Corma, A. From microporous to mesoporous molecular sieve materials and their use in catalysis. Chem. Rev., 1997, 97, 23732419.

[8] Clerici, M. G. Zeolites for fine chemicals production. Topics Catal., 2000, 13, 373-386.

[9] Kresge, C. T.; Leonowicz, M. E.; Roth, W. J.; Vartuli, J. C.; Beck, J. S. Ordered mesoporous molecular sieves synthesized by a liquidcrystal template mechanism. Nature, 1992, 359, 710-712.

[10] Yang, P.; Zhao, D.; Margolese, D. I.; Chmelka, B. F.; Stucky, G. D. Generalized syntheses of large-pore mesoporous metal oxides with semicrystalline frameworks. Nature, 1998, 396, 152-155.

[11] Davis, M. E. Ordered porous materials for emerging applications Nature, 2002, 417, 813-821.

[12] Kapoor, M. P.; Yang, Q.; Inagaki, S. Organization of phenylenebridged hybrid mesoporous silisesquioxane with a crystal-like pore wall from a precursor with nonlinear symmetry. Chem. Mater., 2004, 16, 1209-1213.

[13] Chandra, D.; Mal, N. K.; Mukherjee, M.; Bhaumik, A. Titaniumrich highly ordered mesoporous silica synthesized by using a mixed surfactant system. J. Solid State Chem., 2006, 179, 1802-1807.

[14] Lam, K. F.; Yeung, K. L.; Mckay, G. Selective mesoporous adsorbents for $\mathrm{Cr}_{2} \mathrm{O}_{7}^{2-}$ and $\mathrm{Cu}^{2+}$ separation. Microporous Mesoporous Mater., 2007, 100, 191-201.

[15] Manos, M. J.; Chrissafis, K.; Kanatzidis, M. G. Unique pore selectivity for $\mathrm{Cs}^{+}$and exceptionally high $\mathrm{NH}_{4}{ }^{+}$exchange capacity of the chalcogenide material $\mathrm{K}_{6} \mathrm{Sn}\left[\mathrm{Zn}_{4} \mathrm{Sn}_{4} \mathrm{~S}_{17}\right]$. J. Am. Chem. Soc., 2006, $128,8875-8883$.

[16] Inagaki, S.; Guan, S.; Fukushima, Y.; Ohsuna, T.; Terasaki, O. Novel mesoporous materials with a uniform distribution of organic groups and inorganic oxide in their frameworks. J. Am. Chem. Soc., 1999, 121, 9611-9614.

[17] Melde, B. J.; Holland, B. T.; Blanford, C. F.; Stein, A. Mesoporous sieves with unified hybrid inorganic/organic frameworks. Chem. Mater., 1999, 11, 3302-3308.

[18] Sayari, A.; Hamoudi, S. Periodic mesoporous silica-based organicinorganic nanocomposite materials. Chem. Mater., 2001, 13, 31513168 .

[19] Baleizão, C.; Gigante, B.; Das, D.; Alvaro, M.; Garcia, H.; Corma, A. Synthesis and catalytic activity of a chiral periodic mesoporous organosilica (ChiMO). Chem. Commun., 2003, 1860-1861.

[20] Yamamoto, K.; Sakata, T.; Nohara, Y.; Takahashi, Y.; Tatsumi, T. Organic-inorganic hybrid zeolites containing organic frameworks. Science, 2003, 300, 470-472.

[21] Bhaumik, A.; Kapoor, M. P.; Inagaki, S. Ammoximation of ketones catalyzed by titanium-containing ethane bridged hybrid mesoporous silsesquioxane. Chem. Commun., 2003, 470-471.

[22] Chandra, D.; Yokoi, T.; Tatsumi, T.; Bhaumik, A. Highly Luminescent Organic-Inorganic Hybrid Mesoporous Silicas Containing Tunable Chemosensor Inside the Pore-Wall. Chem. Mater., 2007, 19, 5347-5354.

[23] Yoshitake, H.; Yokoi, T.; Tatsumi, T. Adsorption of chromate and arsenate by amino-functionalized MCM-41 and SBA-1. Chem. Mater., 2002, 14, 4603-4610.

[24] Mal, N.K.; Fujiwara, M.; Tanaka, T. Photocontrolled reversible release of guest molecules from coumarin-modified mesoporous silica. Nature, 2003, 421, 350-353.

[25] Zhang, L.; Qiao, S. Z.; Jin, Y.; Chen, Z. G.; Gu, H. C.; Lu, G. Q. Magnetic hollow spheres of periodic mesoporous organosilica and 
Fe $3 \mathrm{O} 4$ nanocrystals: Fabrication and structure control. Adv. Mater., 2008, 20, 805-809.

[26] Inagaki, S.; Ohtani, O.; Goto, Y.; Okamoto, K.; Ikai, M.; Yamanaka, K. -I.; Tani, T.; Okada, T. Light harvesting by a periodic mesoporous organosilica chromophore. Angew. Chem. Int. Ed., 2009, 48, 4042-4046.

[27] Kimura, T. Synthesis of mesostructured and mesoporous aluminum organophosphonates prepared by using diphosphonic acids with alkylene groups. Chem. Mater., 2005, 17, 337-344.

[28] Sarkar, K.; Laha, S. C.; Bhaumik, A. A new extra large pore silicoaluminophosphate. J. Mater. Chem., 2006, 16, 2438-2444.

[29] Kapoor, M. P.; Inagaki, S.; Yoshida, H. Novel zirconium-titanium phosphates mesoporous materials for hydrogen production by photoinduced water splitting. J. Phys. Chem. B, 2005, 109, 92319238.

[30] Zhao, X. S.; Su, F.; Yan, O.; Guo, W.; Bao, X. Y.; Lv, L.; Zhou, Z. Synthesis and properties of phosphonic acid-grafted hybrid inorganic-organic polymer membranes. J. Mater. Chem., 2006, 16, $858-864$.

[31] Bhaumik, A.; Samanta, S.; Mal, N. K. Highly active disordered extra large pore titanium silicate. Microporous Mesoporous Mater., 2004, 68, 29-35.

[32] Sun, T.; Wong, M. S.; Ying, J. Y. Synthesis of amorphous, microporous silica with adamantanamine as a templating agent. Chem. Commun., 2000, 2057-2058.

[33] Peng, H.; Tang, J.; Yang, L.; Pang, J.; Ashbaugh, H. S.; Brinker, C. J.; Yang, Z.; Lu, Y. Responsive periodic mesoporous polydiacetylene/silica nanocomposites. J. Am. Chem. Soc., 2006, 128, 5304-5305.

[34] Nakajima, K.; Tomita, I.; Hara, M.; Hayashi, S.; Domen, K.; Kondo, J. N. A stable and highly active hybrid mesoporous solid acid catalyst. Adv. Mater., 2005, 17, 1839-1842.

[35] Ravikovitch, P. I.; Neimark, A. V. Density functional theory of adsorption in spherical cavities and pore size characterization of templated nanoporous silicas with cubic and three-dimensional hexagonal structures. Langmuir, 2002, 18, 1550-1560.

[36] Sarkar, K.; Laha, S. C.; Mal, N. K.; Bhaumik, A. A new porous open-framework titanium oxophenylphosphate. J. Solid State Chem., 2008, 181, 2065-2072.
[37] Mustafa, S.; Murtaza, S.; Naeem, A.; Farina, K. Ion exchange sorption of $\mathrm{Pb}^{2+}$ ions on $\mathrm{CrPO}_{4}$. Environ. Tech., 2005, 26, 353-359

[38] Stein Sr., E. W.; Clearfield, A.; Subramanian, M. A. Conductivity of group IV metal sulfophosphonates and a new class of interstratified metal amine-sulfophosphonates. Solid State Ionics., 1996, 83, 113-124.

[39] Schneider, J.; Oliveira, S. L.; Nunes, L. A. O.; Panepucci, H. Local structure of sodium aluminum metaphosphate glasses. J. Am. Ceram. Soc., 2003, 86, 317-324.

[40] Xiang, K. -H.; Pandey, R. A theoretical study of the cluster vibrations in $\mathrm{Cr}_{2} \mathrm{O}_{2}, \mathrm{Cr}_{2} \mathrm{O}_{3}$, and $\mathrm{Cr}_{2} \mathrm{O}_{4}$. J. Phys. Chem. A., 2000, 104, 990-994.

[41] Tarafdar, A.; Biswas, S.; Pramanik, N. K.; Pramanik, P. Synthesis of mesoporous chromium phosphate through an unconventional sol-gel route. Microporous Mesoporous Mater., 2006, 89, 204-208.

[42] Hong, S.; Kim, E.; Kim, D. -W.; Sung, T. -H.; No, K. On measurement of optical band gap of chromium oxide films containing both amorphous and crystalline phases. J. NonCrystalline Solid, 1997, 221, 245-254.

[43] Vinu, A.; Ariga, K.; Mori, T.; Nakanishi, T.; Hishita, S.; Golberg, D.; Bando, Y. Preparation and characterization of well-ordered hexagonal mesoporous carbon nitride. Adv. Mater., 2005, 17, 16481652.

[44] Pawlak, D. A.; Ito, M.; Oku, M.; Shimamura, K.; Fukuda, T. Interpretation of XPS O (1s) in mixed oxides proved on mixed perovskite crystals. J. Phys. Chem. B, 2002, 106, 504-507.

[45] Shevchenko, N.; Zaitsev, V.; Walcarius, A. Bifunctionalized mesoporous silicas for $\mathrm{Cr}(\mathrm{VI})$ reduction and concomitant $\mathrm{Cr}(\mathrm{III})$ immobilization. Environ. Sci. Technol., 2008, 42, 6922-6928.

[46] Fan, X.; Yu, T.; Wang, Y.; Zheng, J.; Gao, L.; Li, Z.; Ye, J.; Zou, Z. Role of phosphorus in synthesis of phosphated mesoporous $\mathrm{TiO}_{2}$ photocatalytic materials by EISA method. Appl. Surf. Sci., 2008, 254, 5191-5198.

[47] Samanta, S.; Mal, N. K.; Bhaumik, A. Mesoporous Cr-MCM-41: an efficient catalyst in selective oxidation of bulky cyclooctane. $J$. Mol. Catal. A. Chem., 2005, 236, 7-11. 